to walk practically, but all could walk after the last joint surgery. Over the course of long-term follow-up the percentage of patients capable of walking decreased: 5 years after the first surgery $92 \%$ could walk, at 10 years $79 \%$ could walk and at 15 years only $60 \%$ could walk. The major causes of this decrease in function were cervical spinal lesions, compression fractures, aggravated pulmonary fibrosis, decreased volition with age, and complications of artificial joints including bone fracture, loosening of implants and deep infection.

At the time of the study, $34 \%$ of patients had already died. In the remaining patients, the survival rate decreased over 15 years, and rapidly so after 10 years. Causes of death included infection, renal failure and interstitial pneumonia indicating rheumatic disease. The disease activity of RA remained high throughout follow-up, and several patients required additional surgery to maintain their activities of daily living.

The investigators conclude that prevention of osteoporosis is essential for improving the outcome of multiple arthroplasty, and that caution against infection and better RA treatment will improve the vital prognosis for multiple arthroplasty patients.

Rachel Murphy

Original article Shinomiya F et al. (2005) Long-term outcome of patients with rheumatoid arthritis treated by multiple arthroplasty. Mod Rheumatol 15: 241-248

\section{Urine-based polymerase chain reaction is not suitable for diagnosing Lyme borreliosis}

Researchers from Germany have shown that, despite developing an optimized protocol, polymerase chain reaction (PCR) analysis of urine is not an acceptable method for diagnosing Lyme borreliosis (LB).

Serologic testing is currently used to confirm a diagnosis of LB, but it has several limitations including serologic cross-reactivity, delayed production of antibodies and an inability to distinguish between ongoing and previous infections. PCR detection of DNA from Borrelia burgdorferi sensu lato- the causative organism of LB-would overcome these limitations, and urine is an attractive sample material because it is easily obtained. PCR analysis of urine samples for diagnosing LB is a controversial issue, however, due to previous conflicting results. Rauter et al. aimed to create an optimized PCR protocol and determine its efficacy in detecting Borrelia DNA and Borrelia organisms in urine.

Urine samples from healthy patients were spiked with whole Borrelia and Borrelia DNA. Investigations demonstrated that spiked urine could be stored for up to 6 months at $-20^{\circ} \mathrm{C}$ without negative effect on spike recovery, that centrifugation had a positive effect on all samples for detecting both DNA and whole Borrelia, and that the inhibition of spike recovery could be attributed to nuclease activity in urine. Taking into account these findings, an optimized, well-controlled and highly sensitive PCR protocol was developed and was used to test urine samples from 12 patients with erythema migrans - an acute infection with Borrelia. Only one patient had a positive PCR result, and the authors conclude that urine is not a suitable material for diagnosing LB.

Rachel Murphy

Original article Rauter C et al. (2005) Critical evaluation of urine-based PCR assay for diagnosis of Lyme borreliosis. Clin Diagn Lab Immunol 12: 910-917

\section{Recombinant osteoprotegerin suppresses bone resorption in juvenile Paget's disease}

Juvenile Paget's disease results from a mutation in the gene encoding osteoprotegerin, a protein that inhibits bone-resorbing osteoclast cells. A study of two adult siblings with juvenile Paget's disease has demonstrated that recombinant osteoprotegerin suppressed bone resorption, increased radial bone mass and decreased skeletal bisphosphonate retention. Both patients also showed radiographic improvement.

The 31-year-old woman and 24-year-old man were both homozygous for an in-frame deletion of nucleotides 638 to 640 in exon 3 of member $11 \mathrm{~B}$ of the superfamily of tumornecrosis-factor receptors (TNFRSF11B). The patients had been treated with intravenous pamindronate 3 years before the current study, but suppression of plasma alkaline phosphatase activity was short-lived and the patients had prominent side-effects.

In the present trial, the patients received an initial dose of $0.1 \mathrm{mg} / \mathrm{kg}$ of subcutaneous recombinant osteoprotegerin, followed by further doses of 